\title{
Impact of dust on solar energy generation based on actual performance
}

\begin{abstract}
Energy and Environment are two important issues in this decade. Using the clean energy such as solar energy generation is growing around word, but with low efficiency. Many parameters from the environment affect the solar photovoltaic panel such as shadow, air pollution and dust. In some experimental setup, there is a small layer that accumulates on top of the photovoltaic (PV). In order to evaluate the effect of dust on Photovoltaic, using two Fix Flat Photovoltaic (FFP) was installed at the Universiti Putra Malaysia. One array is the ñClean arrayò that was cleaned regularly and other is the ñDusty arrayò that was not cleaned during this research. Data was collected at interval each of 30mins from 1st April to 5th December 2013 for both arrays. Power output and Energy yield for both PV arrays are considered. Findings from this research shows that overly power and energy decrease due to the dust, which accumulates on the surface. The contribution this work with other research was done is we are evaluate the power loss in tropical climate which force with many rainy days.
\end{abstract}

Keyword: Component; Fix flat array; P (key words); Photovoltaic generation; PV generation 